\title{
In silico Validation of Expressed Sequence Tags for Alternate Splice Variants of Human myo-inositol Oxygenase Gene
}

\author{
Jayendra Bajracharya $^{1 *}$, Manoj Pradhan ${ }^{2}$, Anju Bajracharya ${ }^{3}$ \\ ${ }^{1}$ Department of Biochemistry, Nepalese Army Institute of Health Sciences \\ ${ }^{2}$ Department of Microbiology, Nepalese Army Institute of Health Sciences \\ ${ }^{3}$ Department of Pharmacy, Little Angels College of Higher Studies, JF Institute of Health Sciences
}

\begin{abstract}
myo-Inositol oxygenase (MIOX) is an enzyme that catabolizes myo-inositol in humans. There is increased MIOX expression and increased MIOX activity in renal tubular cells in diabetic nephropathy. One potential mechanism of increased expression and activity of MIOX is the production of one or more highly stable alternative splice isoform of MIOX transcript. Based on the evidence gathered from the expressed sequence tags (ESTs) associated with MIOX protein sequence, we report that retention of intron 9 in MIOX transcript is a valid alternative splice mechanism. This alternative splice isoform is predicted to have higher stability than the canonical MIOX transcript. Higher stability of the alternative splice variant leads to increased production of MIOX protein isoform with retained structural and potentially, functional features of canonical MIOX protein. Production of this MIOX transcript isoform could be the mechanism of increased expression and activity of MIOX in renal tissues affected by diabetes mellitus. Alteration of the splice mechanism could be a new therapeutic target in prevention and treatment of complications of diabetes mellitus.
\end{abstract}

Keywords: Alternate splice variants, myo-inositol oxygenase, MIOX, expressed sequence tag, In silico validation

\section{INTRODUCTION}

myo-Inositol oxygenase (MIOX) is an enzyme involved in the myo-inositol catabolic pathway in humans [1]. The human MIOX gene is composed of 3538 nucleotides with ten exons and nine introns. The MIOX gene is transcribed to an 855-nucleotide mature transcript. MIOX is expressed mainly in the cells of the proximal convoluted tubule [2]. There is growing evidence that implicate the roles of increased MIOX expression and consequently, its activity in renal tubular cells in pathogenesis of diabetic nephropathy [3]. A variety of factors modulate the expression of a gene. Alternative splicing, however, can modulate the stability of the transcript and ultimately, affect the activity of the protein product [4]. Increase in MIOX expression and activity in renal tubular cells in diabetes may well be related with formation of highly stable isoforms of MIOX transcripts by alternative splicing.

* Correspondence: Jayendra Bajracharya

Department of Biochemistry, Nepalese Army Institute of Health Sciences

E-mail: jayendra.bajrachaya@naihs.edu.np

\begin{abstract}
Alternative splicing of pre-mRNA involves a complex machine called splicesome, which recognizes the donor (GU) and acceptor (AG) splice site signals at 5' and 3'ends of an intron respectively. Once the signals are read, excision of the pre-mRNA occurs at these sites. The excised segment (intron) forms a lariat while the exons are glued together to form a mature mRNA [4]. Alternative splicing can involve exon skipping, complete or partial intron retention, and alternate splice site acceptor or donor at the $5^{\prime}$ or the $3^{\prime}$ ends within any of the exons or introns. Identification of the alternative splice variants of transcripts require examination of database of expressed sequence tags (ESTs), which are short cDNA sequences that represent the expression of a gene in a specific tissue. Detailed analysis of the putative protein products of the alternative splice variants of MIOX transcript is required to validate the ESTs.
\end{abstract}




\section{METHODOLOGY}

The MIOX protein was used as a query sequence for TBLASTN [5] search of ESTs in the database of expressed sequence tag [6] (dbEST) archived in EST divisions of GenBank - the genetic sequence database of National Institute of Health (NIH), European Molecular Biology Laboratory (EMBL), and DNA Data Bank of Japan (DDBJ). The search was carried out on July 16, 2016 at 12:11 AM NST.

Once the ESTs were retrieved, local alignment tools such as CLUSTAL W [7] and Spidey [8] were used to align each of the EST with MIOX gene and its mature transcript for locating the exons, introns, untranslated regions and splice site signals on the ESTs.

Once the alternative splicing events were identified, virtual mRNAs were constructed in silico by incorporating the alternate splice event to the MIOX canonical transcript. The stability of the major alternate splice variant was examined by a program called RNAfold [9]. The secondary structures and the free energies of these variants were also compared to that of MIOX canonical transcript.

These alternate splice variants were translated by using various translation programs such as Transeq and Translator. The predicted protein products were then aligned with the canonical MIOX protein using CLUSTALW. PredictProtein [10] and Swiss Model [11] were used to examine the structure of the predicted protein products.

\section{RESULT}

The TBLASTN search carried out using MIOX protein sequence as a query against dbEST returned 96 hits. Upon careful examination of the 94 hits, there were 75 independent ESTs. Some ESTs were probably repeated in the hits due to alignment of those ESTs with multiple parts of the MIOX protein. Out of the 75 independent ESTs, 53 ESTs were found to have either normal splicing or truncated parts of the mature canonical transcript of MIOX. Twenty-two ESTs showed some form of alternative splice events, which is summarized in Table 1.

Of the different alternative splice events, near complete retention of intron 9 was evident in five independent ESTs. Other splice events were observed in fewer ESTs.

Intron 9 is 103 nucleotides in length. As there are five independent ESTs, near complete or possibly, complete retention of intron 9 is more likely to be a plausible alternative splice event for MIOX gene. The lengths, sources, and percent identities of the ESTs with intron 9 of MIOX gene are shown in Table 2. Based on the higher percent identity with intron 9 of MIOX gene, it can be concluded that retention of intron 9 is a highly likely alternative splice event for MIOX gene.

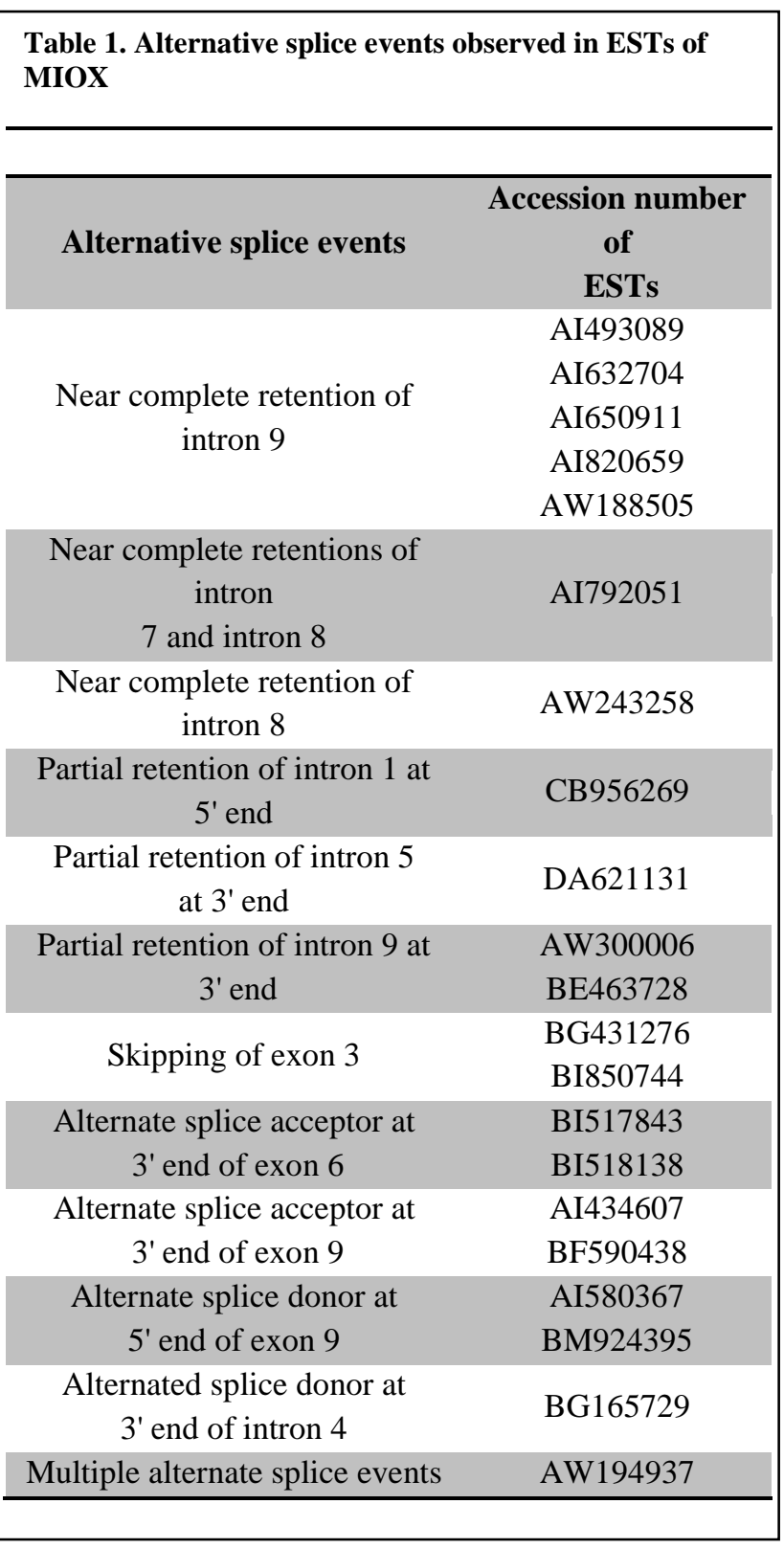

It is evident that the ESTs did not perfectly align with intron 9 sequence throughout its length as shown in Figure 1. This is much more likely due to sequencing error of the transcripts. The presence of very few alternative splice site signals within intron 9 possibly means that the alternative splice variant of $M I O X$ gene most likely would contain the complete sequence intron 9 . 


\begin{abstract}
AI493089 CCCTGGGTGCGGGAGTTCAAGTACGCCCCGCTACCCGCCGAGGGGTGTTG AI632704 CCCTGGGTGCGGGAGTTCAAGTACGCCCCGCTACCCNCCGAGGGGTGTTG

AI650911 CCCTGGGTGCGGGAGTTCAAGTACGC-CCGCTACCCCCCCAAGGGTGTTG AI820659 CCCTGGGTGCGGGAGTCAAGTACGCCCCGCTACCCGCCGAGGGGTGTTG AW188505 CCTTGGGTGCGGGAGTTCAAGTACGCCCCGCTACCCGCCGAGGGGTGTTG

Introng ----------------GTACGCCCCGCTACCCGCCGAGGGGTGTG

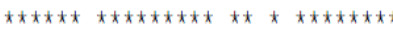

AI493089 TGGGAGTGAAAAGGGGGTTGGGTGGGGGGCCTGGGGGTT--CACGGGG AI632704 TGGGAGTGAAAAAAGGGTTGGGTGGGGGGCCTGGGGGTTTTTCACG-GG AI650911 TGGGAGTGAAAAAAGGGGTTGGGTGGGGGGC-TGGGGGTTT--CACNGGG AI820659 TGGGAGTGAAAG-GGGGGTTGGGTGGGGGGCCTGGGGGTTTCTCACG-GG AW188505 TGGGAGTAAAAGAGGGGTTGGGTGGGGGGCCTGGGGTTCTTCACG-GG Introng TGGGAGTGAAGAGGGGGTTGGGTGGGGGCCTGGGGGTTCTCACG-GG

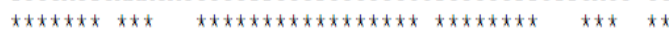

AI493089 GCTC-CCGCCCCCTCCCTGCTGCAGCAAGTTCGACCTCTACACCAAGTGC AI632704 GCTCACCNGCCCCTCCCTGCTGCAGCAAGTTCGACCTTTACACCAAGTGC AI650911 GCTC-CCGCCCCTTCCTGNTGCAGCAAGTTCGACCTCTACACCAAGTGC AI820659 GCTCA-CCGCCCCTCCCTGCTGCAGCAAGTTCGACCTCTACACCAAGTGC AW188505 GCTCA-CCGCCCCTCCCTGCTGCAGCAAGTTCGACCTCTACACCAAGTGC

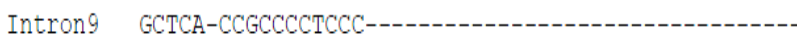

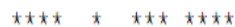

Figure 1. Multiple sequence alignment of the ESTs with near complete retention of intron 9 of MIOX gene as aligned with the intron 9 sequence

Moreover, the secondary structure of in silico generated virtual mRNA constructed by incorporating the complete sequence of intron 9 was found to be similar to the secondary structure of MIOX canonical transcript as shown in Figure 2.

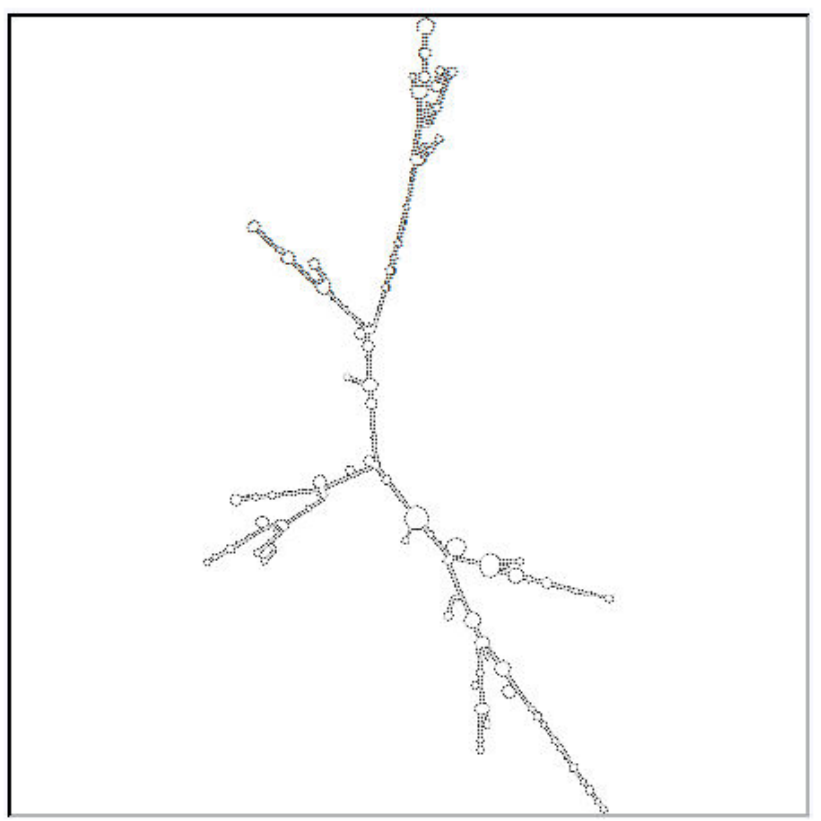

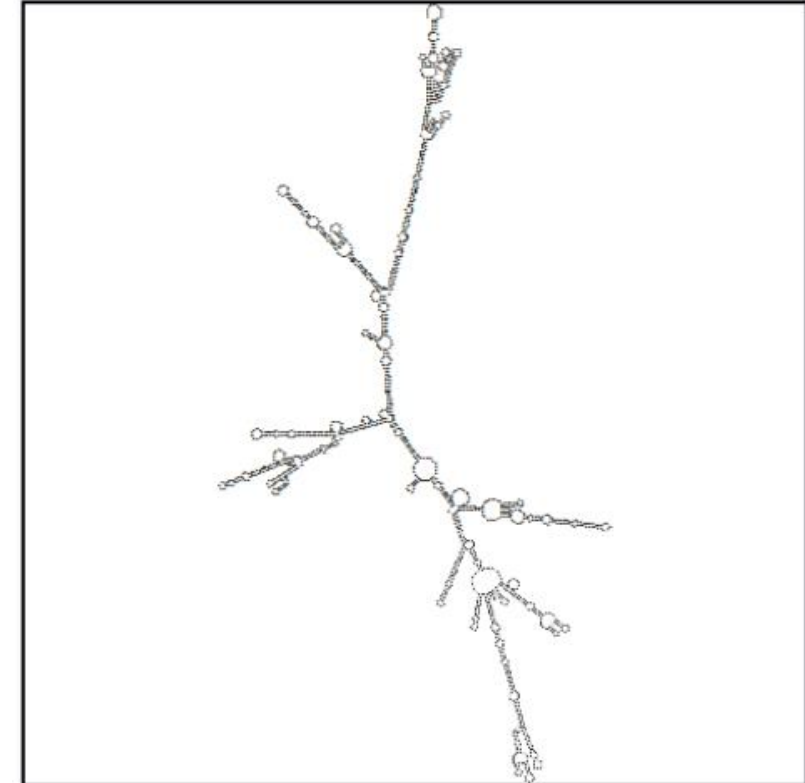

Figure 2. Secondary structures of canonical transcript of MIOX (top) and the putative transcript of MIOX with intron 9 retention (bottom).

Free energies of the mRNA secondary structures determine their stabilities. Free-energy calculations of the ESTs and native transcript show that native mRNA sequences have more negative free energy than randomized mRNA sequences with similar nucleotide compositions (Seffens and Digby, 1992). As predicted by the RNAfold program, the intron 9 complete retention splice variant mRNA has the most negative free energy $(-725.15 \mathrm{kcal} / \mathrm{mol})$ while the MIOX canonical transcript has a negative free energy ($655.90 \mathrm{kcal} / \mathrm{mol}$ ). Thermodynamically, low free energy means higher stability. So, the alternative splice variant with complete incorporation of intron 9 in MIOX mRNA is highly likely to be stable, and thus, may be transcribed to a functional protein.

In silico translation of the alternative splice variant with complete incorporation of intron 9 in MIOX mRNA produced a protein product of 304 amino acids. As the numbers of nucleotides added to make these splice variants is 103, which is not a multiple of three, frame-shift is predicted to occur downstream of this sequence. The translated product retains high identity to the canonical MIOX protein and also retains all the secondary structures of canonical MIOX protein. Modeling of the active site of the translated product shows the retention of the MIOX active site in this protein as shown in Figure 3. 

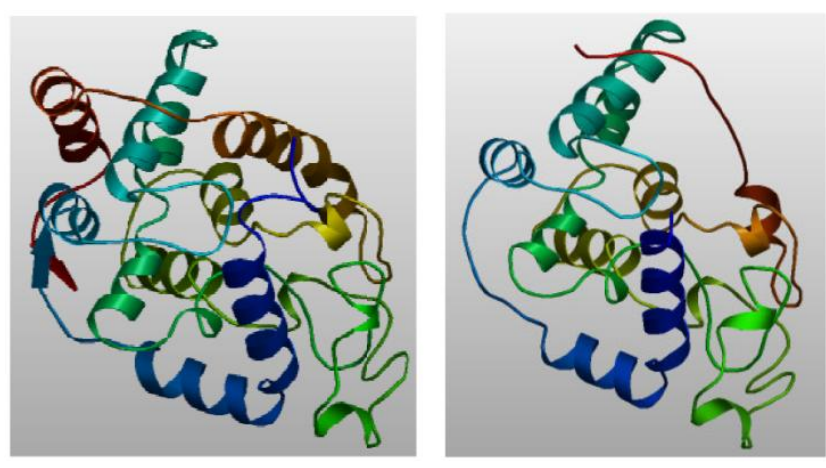

Figure 3. Homology modeling of MIOX protein isoform produced from retention of intron 9 is shown on the right. Canonical human MIOX protein shown on the left is used as a template to model the MIOX protein isoform.

It has been shown that the activity of the native MIOX protein depends on Asp35, Lys127, the inositol binding pocket, and the lid that stabilizes the substrate to the inositol binding pocket [12]. Surprisingly, the translated product of the virtual mRNA of MIOX with complete retention of intron retains all of the above important amino acids that are required for structural stability and enzymatic activity of MIOX. Thus, MIOX protein encoded by mRNA with retention of intron 9 may be physiologically relevant.

\section{DISCUSSION}

This study establishes protein product of the alternate splice variant of MIOX transcript with complete retention of intron 9 as a potentially functional protein. The thermodynamically stable nature of the intron 9 retained transcript could result in its higher rates of translation to a protein product, which appears to be structurally similar to that of the MIOX canonical protein.

Several studies have shown increased activity of MIOX in tissues affected by diabetes mellitus [13,14]. One of the potential causes of increased MIOX activity is production of highly stable alternate splice variant of MIOX transcript. Intron retention has been described as one of the common mechanism of production alternate splice variants. It is highly likely that the MIOX transcript with retention of intron 9 could be the 'culprit' transcript, which may potentially lead to increased activity of MIOX in tissues affected by diabetes mellitus. Promotion of alternative splicing of transcript of other genes in diabetes mellitus has been reported [15].
The molecular alterations and mechanisms that lead to retention of intron 9 in MIOX transcript is an area that needs to be explored. Isolation of the protein product of the splice variant is needed to confirm the in vivo existence of the splice variant. Assays for enzyme activity will also be needed to determine the actual enzymatic activity of the protein product. If in vitro and in vivo studies prove that the retention of intron 9 in MIOX transcript leads to increased MIOX activity in tissues affected by diabetes mellitus, this could direct the current research to development of a new therapeutic strategy on preventing and/or treating the complications of diabetes mellitus.

\section{REFERENCES}

1. Hankes LV, Politzer WM, Touster O, Anderson L. Myoinositol catabolism in human pentosurics: The predominant role of the glucuronate-xylulose-pentose phosphate pathway. Ann N Y Acad Sci 1969 Oct 17;165(2):564-76.

2. Arner RJ, Prabhu KS, Reddy CC. Molecular cloning, expression, and characterization of myo-inositol oxygenase from mouse, rat, and human kidney. Biochem Biophys Res Commun 2004 Nov 26;324(4):1386-92.

3. Arner RJ, Prabhu KS, Krishnan V, Johnson MC, Reddy CC. Expression of myoinositol oxygenase in tissues susceptible to diabetic complications. Biochem Biophys Res Commun 2006 Jan 20;339(3):816-20.

4. Fedor MJ. Alternative splicing minireview series: Combinatorial control facilitates splicing regulation of gene expression and enhances genome diversity. J Biol Chem 2008 Jan 18;283(3):1209-10.

5. http://blast.ncbi.nlm.nih.gov/Blast.cgi

6. http://www.ncbi.nlm.nih.gov/dbEST/

7. http://www.ebi.ac.uk/Tools/clustalw2/index.html

8. http://www.ncbi.nlm.nih.gov/spidey/

9. http://www.tbi.univie.ac.at/RNA/RNAfold.html

10. http://www.predictprotein.org/

11. http://swissmodel.expasy.org/SWISS-MODEL.html

12. Thorsell AG, Persson C, Voevodskaya N, Busam RD, Hammarstrom M, Graslund S, Graslund A, Hallberg BM. Structural and biophysical characterisation of human myoinositol oxygenase. J Biol Chem 2008 Mar 24.

13. Nayak B, Xie P, Akagi S, Yang Q, Sun L, Wada J, Thakur A, Danesh FR, Chugh SS, Kanwar YS. Modulation of renalspecific oxidoreductase/myo-inositol oxygenase by highglucose ambience. Proc Natl Acad Sci U S A 2005 Dec $13 ; 102(50): 17952-7.101$ 
14. Prabhu KS, Arner RJ, Vunta H, Reddy CC. Up-regulation of human myo-inositol oxygenase by hyperosmotic stress in renal proximal tubular epithelial cells. J Biol Chem 2005 May 20;280(20):19895-901.

15. Davies KP, Zhao W, Tar M, Figueroa JC, Desai P, Verselis VK, Kronengold J, Wang HZ, Melman A, Christ GJ.

Diabetes-induced changes in the alternative splicing of the slo gene in corporal tissue. Eur Urol 2007 Oct;52(4):122937.

\section{Article History:}

Received: 25 July, 2016

Accepted: 15 September, 2016

Published online: 5 October, 2016

\section{For Citation:}

Bajracharya J, Pradhan M, Bajracharya A. In silico Validation of Expressed Sequence Tags for Alternate Splice Variants of Human myo-inositol Oxygenase Gene. International Journal of Medicine \& Biomedical Sciences. 2016; 1(4):1721 